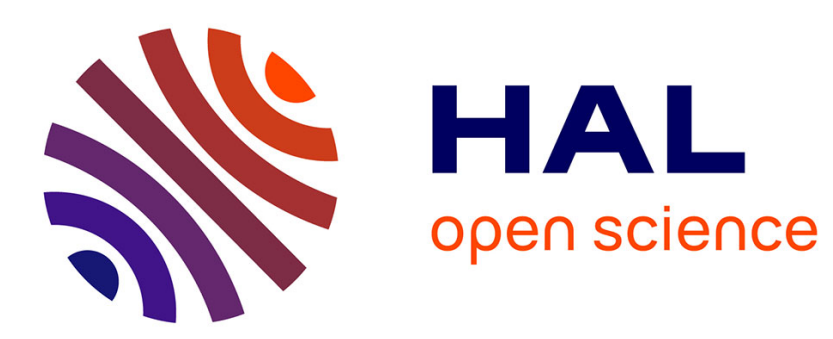

\title{
La participation des usagers de la Mobile TV aux formes visuelles de la coordination sociale.
}

Julien Figeac

\section{To cite this version:}

Julien Figeac. La participation des usagers de la Mobile TV aux formes visuelles de la coordination sociale.. Les Cahiers du numérique, 2010, 6 (2), pp.41-64. halshs-00602170

\section{HAL Id: halshs-00602170 \\ https://shs.hal.science/halshs-00602170}

Submitted on 21 Jun 2011

HAL is a multi-disciplinary open access archive for the deposit and dissemination of scientific research documents, whether they are published or not. The documents may come from teaching and research institutions in France or abroad, or from public or private research centers.
L'archive ouverte pluridisciplinaire HAL, est destinée au dépôt et à la diffusion de documents scientifiques de niveau recherche, publiés ou non, émanant des établissements d'enseignement et de recherche français ou étrangers, des laboratoires publics ou privés. 
LA PARTICIPATION DES USAGERS DE LA MOBILE TV AUX FORMES VISUELLES DE LA COORDINATION SOCIALE

Julien Figeac 
$2 \mathrm{LCN} \mathrm{n}{ }^{\circ} / 2010$. Usages, pratiques et ergonomie des nouveaux supports numériques

Les téléphones multimédia de dernière génération permettent de transporter de nombreuses pratiques médiatiques, habituellement cloisonnées dans la sphère domiciliaire, au sein des situations de mobilité. La Mobile TV s'inscrit au cœur de cette tendance et a fait l'objet de nombreuses études ayant suivi trois directions.

Durant la mise en place de prototypes de services, les travaux sur l'ergonomie des interfaces ont tout d'abord questionné l'interactivité de la Mobile TV et de son support numérique (Södergard, 2003). Par exemple, la multifonctionnalité des téléphones mobiles demandait de produire une interface permettant de bifurquer sans difficulté entre les différentes fonctions multimédia (Lehtola, Mokka, 2002) tout en laissant ces utilisations perméables aux alertes et aux notifications émises par les fonctions communicationnelles pour que l'utilisateur puisse maintenir sa joignabilité.

Cette entrée par l'ergonomie des interface a ensuite été associée à une étude plus générale des modes de consommation de la Mobile TV. L'objectif étant de mettre en place une offre de contenus qui soit en phase avec les attentes des utilisateurs. Un débat a ainsi eu lieu sur ces attentes et sur les motivations d'usage inscrites au principe de cette pratique télévisuelle nomade. D'un côté, certains défendent l'idée que l'ennui, éprouvé notamment dans les transports en commun, forme la motivation première des «usages privés» (Södergard, 2003) de ce service ; ces derniers se distinguant des «usages partagés » entre deux co-récepteurs ou plus. D'autres invalident la possibilité d'élever l'ennui au rang de motivation d'usage (O'Hara et al. 2007) tout en considérant que les différents contenus consultables en situation de mobilité invitent l'utilisateur à affirmer des préférences même s'il s'est tourné vers ce service par ennui (Figeac, 2010).

La troisième entrée vise à saisir les effets des contextes sur les usages en comparant notamment les utilisations domiciliaires avec celles réalisées dans l'espace public (Oksman et al., 2007). Il a ainsi pu être montré comment les utilisateurs bifurquent d'un média à l'autre en fonction de l'intrusivité, dans leur sphère d'écoute, des bruits et des mouvements extérieurs: ils privilégient certains médias (livres, journaux, Internet) quand l'ambiance est «calme » et d'autres (écoute de la musique) dans les environnements «bruyants» (Södergard, 2003). Et, ils visionnent la Mobile TV en toute situation mais privilégient les «News» quand il y a du bruit autour d'eux. Cette troisième entrée va de paire avec une analyse de la participation des usagers à la vie publique. Ainsi, malgré ces désagréments sonores des environnements urbains, cet écran de poche permet à l'utilisateur d'instaurer une relation intimiste avec ses préférences télévisuelles (Chipchase et al., 2007). Comme il est difficile de s'installer à plusieurs autour de cet écran et comme sa réception en situation de 
$3 \mathrm{LCN} \mathrm{n}{ }^{\circ} / 2010$. Usages, pratiques et ergonomie des nouveaux supports numériques

mobilité est plutôt solitaire, la Mobile TV alimente l'idée d'un repli des utilisateurs dans une bulle intimiste, d'usages égocentrés désolidarisés de l'environnement social immédiat.

Dans le cadre de cette contribution, nous souhaitons documenter ce dernier aspect des usages de la Mobile TV en décrivant comment l'engagement perceptif, auditif et visuel, dans cette réception télévisuelle nomade vient renouveler les formes de la coordination sociale dans l'espace public. Cette problématique de recherche reste peu explorée. Pour la développer, nous devons maintenant faire un détour par les « déambulations spatio-phoniques » des « auditeurs-baladeurs » (Thibaud, 1994).

\section{L'encastrement de la réception télévisuelle et des formes simples de la coordination sociale}

A travers une ethnographie des usages du walkman, il a été démontré que l'écoute musicale dans les lieux publics donne rarement lieu à un repli total dans une bulle intimiste dans la mesure où les auditeurs-baladeurs règlent le volume sonore afin que les sonorités diffusées par les oreillettes restent perméables aux bruits des environnements urbains (Thibaud, 1994), notamment aux indications sonores permettant de conduire et d'optimiser le déplacement (Pecqueux, 2009). Il est dès lors difficile de renvoyer cette pratique musicale à un individualisme forcené. Si ces utilisateurs se désolidarisent partiellement de l'environnement sonore perçu par leurs co-présents, ils habitent en revanche le même espace visuel. Cette pratique musicale laisse dès lors la porte ouverte à des démonstrations de politesse et de courtoisie, orchestrées par des orientations de regards et des mouvements corporels, comme c'est par exemple le cas lorsque quelqu'un détourne ses jambes de l'allée d'une rame du métro pour libérer l'accès à une place assise. Cette orchestration de la civilité renvoie ici à un choix individuel car il est difficile de faire du walkman un intermédiaire technologique poussant à se substituer aux attentes de coordination publicisées par les autres passagers.

Ceci dit, en va-t-il de même avec la Mobile TV? Pour visionner un programme télévisé, ce dispositif médiatique invite l'utilisateur à porter des écouteurs et à rester le regard dirigé en direction de l'écran. A la différence de la lecture, ce support numérique peut davantage inciter les usagers à se couper de leur environnement immédiat en les dissuadant de rester attentifs aux sollicitations formulées par les autres passagers. En des termes plus polémiques, la réception télévisuelle sur les dispositifs mobiles rend-elle les usagers plus égoïstes, accentue-t-elle cette inclination des téléphonistes au "détachement» 
de la situation vécue pouvant être vécu par les co-présents « comme un déni, et donc entraîner une réaction négative » (Jauréguiberry, 1998) ?

L'objectif de cette contribution consiste à rendre compte de la manière dont l'engagement perceptif dans cette pratique télévisuelle vient affecter les modalités de participation aux "formes simples de la coordination sociale» (Conein, 2005). Et réciproquement, nous décrirons comment les usages de la Mobile TV et l'engagement perceptif dans cette réception télévisuelle nomade sont informés par ces formes de la coordination sociale.

Pour répondre à ces questions, nous nous appuierons sur des enregistrements vidéos des usages de la Mobile TV réalisés, à l'aide de lunettes caméra, par 15 utilisateurs durant leurs déplacements quotidiens entre leur domicile et leur lieu de travail. Ce dispositif méthodologique va nous permettre de décrire les orientations de regards des usagers et, par là-même, de rendre compte de l'organisation de l'activité poursuivie, telle qu'elle est descriptible et intelligible en suivant l'orientation des « coups d'œil » et des « regards dirigés » (Sudnow, 2002) capturée par les lunettes caméra.

L'analyse de ces données pose problème car il est difficile de prouver que ce qui apparait au centre du champ de vision de l'usager est effectivement au cœur de son attention. Cependant, comme les utilisateurs restent focalisés sur l'écran de la Mobile TV, il est possible de décrire en détail les moments où ils se désengagent visuellement de l'écran du téléphone pour regarder autour d'eux, en adressant de brefs coups d'œil aux co-présents ou des regards dirigés vers les repères du déplacement. Il devient ainsi possible de décrire comment ils bifurquent d'une activité à l'autre, de la réception télévisuelle à la conduite de leur déplacement, et comment la conduite de ces activités les amène à se coordonner, visuellement et à toute fin pratique, avec les co-présents. C'est du moins l'hypothèse que nous défendons en considérant, dans la continuité des travaux ethnométhodologiques, que la régulation des usages de la Mobile TV dans les lieux publics ne peut être renvoyée à l'efficience supposée de normes de conduite, externes et inflexibles, mais fait l'objet d'une négociation entre interactants, en situation, en fonction des activités qu'ils poursuivent respectivement (lire, écouter de la musique, attendre le terminus, etc.).

Pour décrire l'encastrement des usages de la Mobile TV dans les formes simples de la coordination sociale, nous exploiterons, parmi les 80 heures d'enregistrements de notre base de données, la singularité d'un des parcours filmés car il est susceptible de concorder avec cette démarche probatoire. Durant ce trajet, la participante va être contactée par son compagnon à un moment peu propice qui l'amène à clore instantanément cette conversation par: "je te rappelle tout à l'heure quand je suis à l'aise ». Nous allons dès lors décrire 
$5 \mathrm{LCN} \mathrm{n}{ }^{\circ} / 2010$. Usages, pratiques et ergonomie des nouveaux supports numériques

son engagement visuel dans cette activité, qu'elle clôture car elle n'est pas à l'aise, afin de le comparer avec celui qu'elle va adopter une fois qu'elle sera, de fait, en situation d'« être à l'aise » pour rappeler son correspondant. Ce détour va nous permettre de comparer cette orientation visuelle dans une conversation téléphonique avec celui déployé, quelques minutes après dans ce même contexte, pour visionner la Mobile TV. Cette comparaison nous permettra notamment de référer nos descriptions aux travaux documentant, en s'inspirant de la proxémique (Hall, 1966), l'agencement interactionnel et spatial des conversations dans les lieux publics (Kendon, 1990, Morel, 2002), notamment dans les wagons des transports en commun (Murtagh, 2001).

\section{L'agencement interactionnel et spatial de l'« être à l'aise "}

Sur la base de la retranscription d'un trajet d'une participante, nous allons décrire comment elle s'installe dans un usage de la Mobile TV durant ce trajet entre son domicile et son lieu de travail. Pour regarder un programme télévisé le plus rapidement possible, une fois installée dans le RER, elle se connecte au service dès qu'elle se trouve sur le quai. Ce jour-là, lorsqu'elle monte dans la rame en conservant ses écouteurs et son téléphone multimédia en main, elle n'a plus qu'à appuyer sur une touche pour pouvoir regarder la chaîne M6. Ces « gestes préparatoires » (Datchary, Licoppe, 2007) réalisés sur le quai visibilisent une anticipation de l'orientation future de son activité médiatique, une fois que celle-ci ne sera plus subordonnée à la conduite de cette phase de micromobilité. Cependant, un appel entrant va venir parasiter cette anticipation.

En effet, à travers ce geste préparatoire consistant à conserver en main son téléphone activé, elle maintient également sa joignabilité téléphonique du fait de la multifonctionnalité de ce dispositif. C'est ce qui se passe au moment où la sonnerie retentit $\left(02.14 .03^{1}\right)$. A ce moment là, elle s'apprête à s'asseoir (cf. Photo 1) et jette alors immédiatement un coup d'œil en direction de son téléphone (02.14.18; cf. Photo 2).

\footnotetext{
${ }^{1}$ La temporalité de la retranscription est exprimée en : Minutes.Secondes.Centisecondes. Cette retranscription ne peut pas être reproduite ici dans la mesure où elle s'étend sur une trentaine de pages.
} 
$6 \mathrm{LCN} \mathrm{n}{ }^{\circ} / 2010$. Usages, pratiques et ergonomie des nouveaux supports numériques

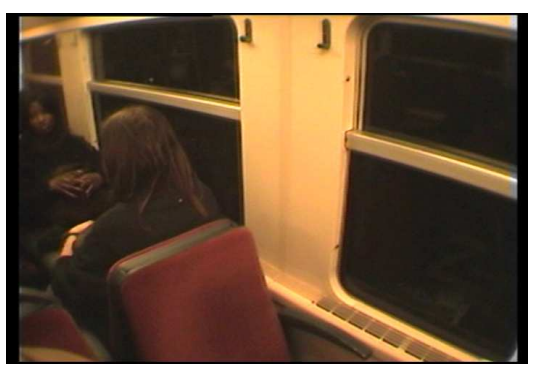

Photo 1 : La sonnerie retentit

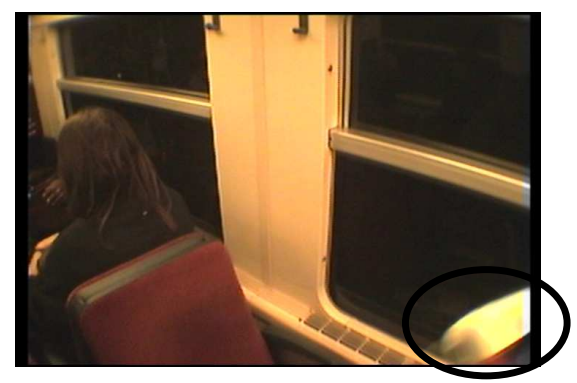

Photo 2 : Coup d'xil sur l'appel entrant

La brièveté de ce mouvement, qui dure quelques centièmes de secondes, lui a probablement laissé le temps de lire le nom de l'appelant. S'il n'est pas possible de statuer sur ce fait, il apparaît en revanche qu'elle a de fait visibilisé, aux yeux des co-présents, qu'elle était la destinataire de cet appel.

Il est intéressant de noter qu'une fois assise (02.19.03), elle va instantanément faire un mouvement de tête vers la droite. Durant ce mouvement, elle va établir un premier contact visuel avec l'homme situé face à elle (02.18.23 ; cf. Photo 3). Il ne regarde pas directement dans sa direction dans la mesure où il finit de positionner son sac à dos sur le siège limitrophe. Elle prolonge ce mouvement de tête tout en commençant à dire «Pardon» (02.19.16) ; excuses qu'elle clôt quelques centièmes de secondes après, en même temps que son mouvement de tête (02.19.25; cf. Photo 4). Elle a dès lors adressé ses excuses sans maintenir un regard dirigé vers l'un de leurs deux principaux destinataires.

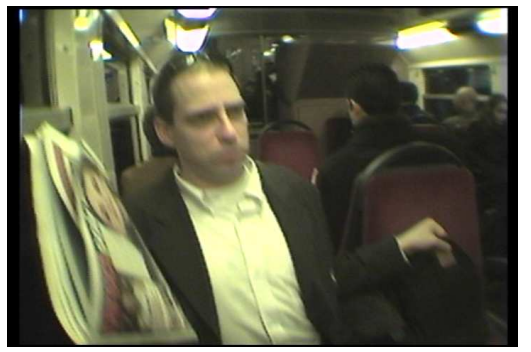

Photo 3 : Premier contact visuel

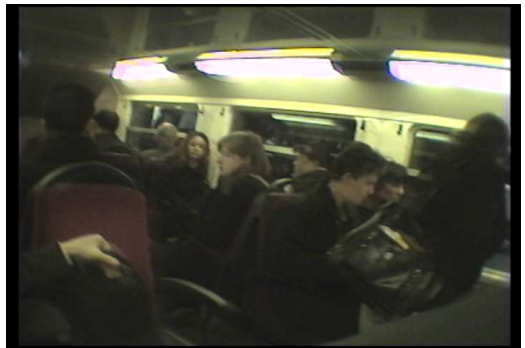

Photo 4 : Orientation du «Pardon »

Cette description nous permet, a minima, de préciser que le comportement de cette participante ne peut pas être renvoyé à la supposée arrogance des téléphonistes qui dérogeraient aux règles de civilité pour maintenir leur joignabilité. En effet, elle ne répond (02.22.06) qu'après avoir formulé ses excuses (02.21.11) juste avant que l'homme situé face à elle regarde dans sa 
direction (cf. photo $5 ; 02.21 .14$ ). Pour répondre, elle tourne la tête vers la gauche, l’incline légèrement, puis dit « oui » (02.22.06; cf. photo 6$)$.

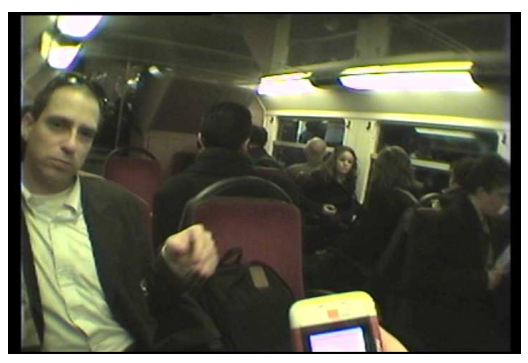

Photo 5. La participante décroche

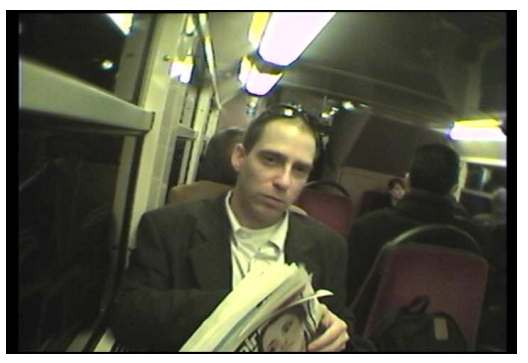

Photo 6. Elle répond

Il n'est pas nécessaire ici d'analyser en détail cette conversation téléphonique. Repérons simplement l'orientation visuelle de la participante pendant cet appel. Même s'il n'est pas possible de savoir où est-ce qu'elle dirige son regard, on peut noter que sa tête est orientée droit devant elle, en direction de l'homme assis en face (cf. Photo 6). Autrement dit, elle n'entretient pas cette conversation téléphonique en regardant, stratégiquement selon Murtagh, vers un «espace neutre » (Murtagh, 2001) qui lui permettrait d'éviter de croiser le regard de cet homme.

Cette orientation visuelle peut être référée avec ce qu'elle dit ensuite (02.24.19) à son interlocuteur distant : "Heu, oui ! Mais tu sais je te rappelle tout à l'heure quand je suis à l'aise ». Une fois ce tour de parole clôt (02.27.11), et une fois que sa proposition a pu être validée par son correspondant, par exemple par un « D'accord», elle clôt cette conversation par : «A t'à l'heure » (02.28.06). Elle annonce ainsi à son interlocuteur qu'elle ré-ouvrira cette conversation une fois qu'elle sera à l'aise. L'intrusivité des conversations téléphoniques dans les wagons des transports en commun pousse souvent les téléphonistes à clore ainsi les appels entrants en repoussant la conversation à plus tard (Murtagh, 2001). La séquence traitée ici se démarque de cette tendance dans la mesure où cette téléphoniste va malgré tout rappeler son correspondant. Autrement dit, cette séquence montre que, quelle que soit l'acceptabilité des usages du téléphone mobile dans ce lieu public, une tendance plus générale prévaut: la recherche d'un compromis entre les téléphonistes et les co-présents à travers des aménagements interactionnels (Morel, 2002). L'objectif consiste dès lors à décrire comment la participante va redéfinir cette situation pour négocier ce compromis et agencer cet état d'« être à l'aise».

Pour amorcer cette description, il convient de partir du moment où elle a dit à l'appelant être dans une position inconfortable. Juste après avoir publicisé cet état (02.27.11), l'homme situé face à elle a cessé de regarder fixement dans sa direction en réinvestissant visuellement son quotidien (02.27.19; cf. Photo 7). 
$8 \mathrm{LCN} \mathrm{n}{ }^{\circ} / 2010$. Usages, pratiques et ergonomie des nouveaux supports numériques

A travers le maintien de ce « regard dirigé » (Sudnow, 2002), ce passager a de fait confronté la participante à la gêne qu'elle occasionnait par la réception de cet appel. Ce regard peut donc être traité comme une action (Sacks, 1992) car il manifeste un sentiment (être dérangé) et un jugement normatif (désapprouver) ou, du moins, il a pu être interprété comme tel. Cet interactant y met donc un terme une fois que la participante annonce vouloir reporter cette conversation. Puis, le fait qu'elle réitère ses excuses (02.30.16), juste après avoir raccroché (02.28.29), lui permet de tenter de réparer le dérangement occasionné. Elle les exprime au moment où elle clôt son mouvement de tête vers la droite, quand l'homme assis à côté d'elle entre dans son champ de vision (02.30.16 ; cf. Photo 8).

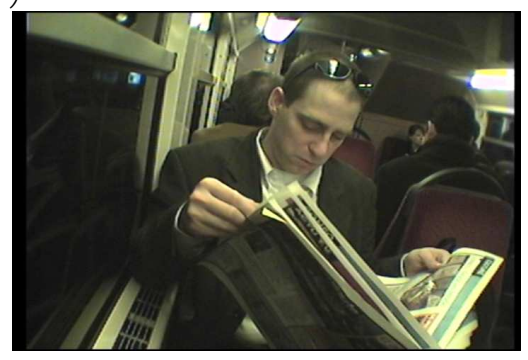

Photo 7. Reprise d'une activité focalisée

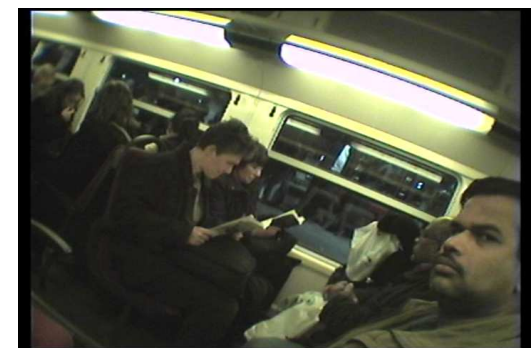

Photo 8. Orientation de l' "Excusez-moi"

Elle amorce la phase suivante, durant laquelle elle va agencer son « être à l'aise », par saisir le sac plastique (02.31.18) qu'elle avait déposé sur ses jambes, avant de se soulever de son siège (02.34.06) et de se rasseoir (02.37.27). A ce moment là, elle pose son sac à main entre sa cuisse et la paroi de la rame du RER, puis repositionne le sac plastique sur ses jambes (cf. Photo 9). Immédiatement après, elle prend en main son téléphone (02.38.26) et ses écouteurs (02.41.01). Suite à l'appel entrant, son téléphone a réaffiché la page d'accueil de la Mobile TV (cf. Photo 10). Il réactualise ainsi son geste préparatoire déployé sur le quai pour optimiser la réception de la chaîne M6.

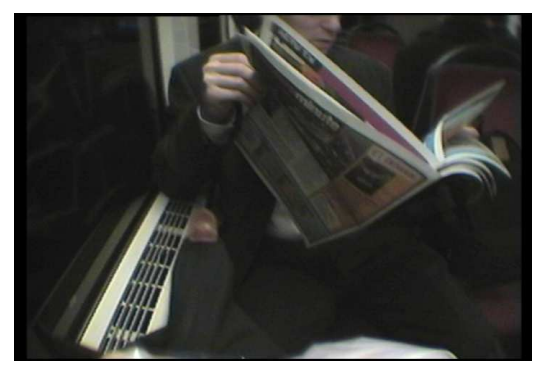

Photo 9. Réajustement de la position assise

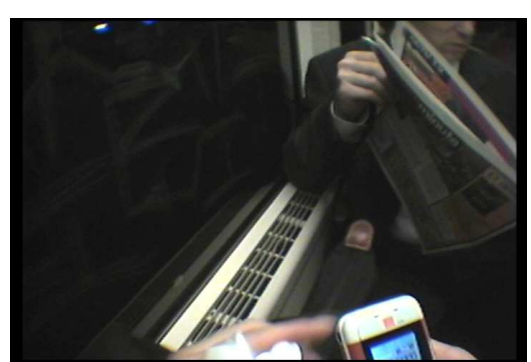

Photo 10. Réactualisation du geste préparatoire 
$9 \mathrm{LCN} \mathrm{n}{ }^{\circ} / 2010$. Usages, pratiques et ergonomie des nouveaux supports numériques

Une fois qu'elle a branché et démêlé le câble des écouteurs, elle positionne l'oreillette droite (03.26.13), puis initie un appel téléphonique (03.28.16) avant de positionner l'oreillette gauche (03.32.06). Comme elle rappelle son correspondant à ce moment là, nous pouvons en déduire qu'elle est maintenant suffisamment à l'aise pour ré-ouvrir cette conversation téléphonique. Pour y parvenir, elle s'est donc excusée, levée et assise tout en positionnant différemment ses sacs, et elle a mis ses oreillettes. Ces dernières jouent la fonction de « kit main libre » et ont de fait été associées aux éléments qu'elle a agencés pour « être à l'aise ». Nous pouvons également noter qu'elle a rappelé son correspondant une fois que le RER évoluait en surface (cf. Photo 10). S’il n'est pas possible de préjuger de l'importance de cet aspect, il va apparaitre comme central dans la mesure il décloisonne l'espace perceptif partagé.

En effet, c'est au dernier moment (03.46.07), juste avant d'engager la conversation par «C'est moi Khaled» (03.48.11), qu'elle va tourner sa tête vers la gauche, vers l'extérieur (cf. Photo 11). Après cette ouverture, elle va prolonger cette orientation du regard vers cet «espace neutre 》 (Murtagh, 2001) (03.51.01 ; cf. Photo 12) avant de prolonger la discussion en disant «Ouai» (03.52.45). En orientant ainsi son regard, elle peut entretenir cette conversation sans croiser les regards des autres passagers, sans être confrontée à d'éventuels signes visuels de désapprobation. Elle manifeste aussi aux co-présents son indisponibilité temporaire à répondre à leurs éventuelles sollicitations (Murtagh, 2001). Dès lors, ce positionnement corporel et cette orientation stratégique du regard vers un "espace neutre » entrent ici dans l'agencement lui permettant d'« être à l'aise » pour entretenir cette conversation téléphonique.

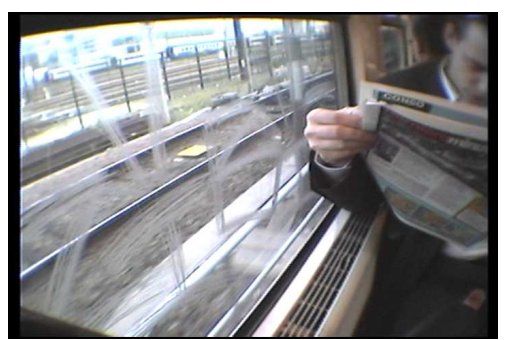

Photo 11. Regard vers un espace neutre 1

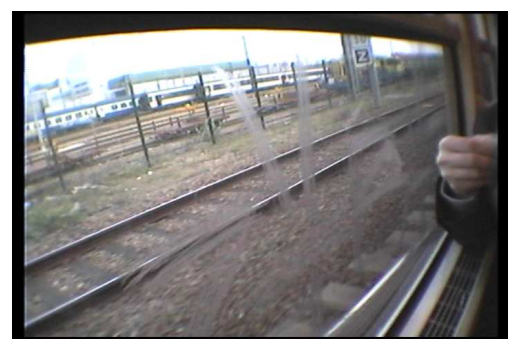

Photo 12 : Regard vers un espace neutre 2

Peu après, sa conversation est interrompue par la perte du réseau téléphonique. Elle regarde alors son téléphone qui affiche l'écran de veille (04.00.21 ; cf. Photo 13). Nous avions vu précédemment que son téléphone était paramétré pour revenir directement sur la Mobile TV une fois un appel entrant clôt (02.41.07). C'est ainsi que ce dispositif a pu mémoriser le geste préparatoire réalisé lorsqu'elle était encore sur le quai de la station du RER. Il n'en va plus de même ici puisqu'en rappelant son correspondant, elle a 
$10 \mathrm{LCN} \mathrm{n}^{\circ} / 2010$. Usages, pratiques et ergonomie des nouveaux supports numériques

désactivé ce service. Elle doit donc reprendre sa navigation à zéro pour regarder la chaîne M6, ce qui lui prendra environ 2 minutes.

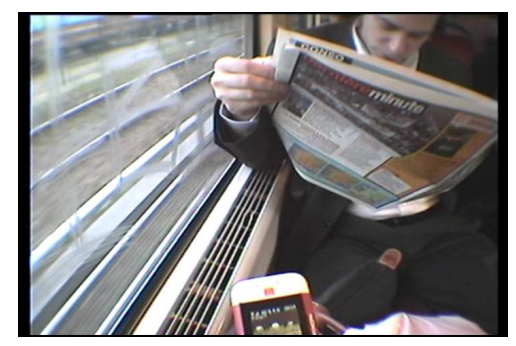

Photo 13 : Interruption de la conversation

La description de cette séquence s'est avérée nécessaire car elle nous permet de tirer plusieurs constats. Lors des entretiens, tous les utilisateurs ont reconnu suspendre systématiquement leurs usages des fonctions multimédia pour privilégier la réponse aux messages entrants. Autrement dit, l'attachement à un contenu médiatique ou un programme télévisé n'est pas suffisamment fort pour justifier la mise entre parenthèse de la joignabilité. Tout en considérant, comme cette séquence le montre, que ce lien de subordination est inscrit dans la configuration même des téléphones mobiles.

En mettant en place des stratégies pour optimiser la consultation de la Mobile TV, les utilisateurs main-tiennent un usage potentiellement intrusif car il peut être interrompu par la propagation dérangeante de la sonnerie téléphonique. Cette intrusivité reste bien réelle. Même si la participante n'a pas été interpellée verbalement par un autre passager, elle s'est vu adresser un « regard dirigé » suffisamment prolongé et insistant pour l'inciter à clore sa conversation. Cette séquence nous permet donc de tirer une généralité quant à l'acceptation des usages du téléphone mobile dans les lieux publics. Les usages «bruyants» restent sujets à désapprobation dans les transports en commun. Et les utilisateurs se sentent suffisamment "mal à l'aise", face la gêne qu'ils occasionnent, pour s'excuser. Ceci dit, ce ressenti se mesure à la hauteur de la gêne occasionnée car c'est bien le bruit strident de la sonnerie téléphonique qui a fait perdre la face à cette utilisatrice. Il lui a suffit d'adresser ses excuses et de se positionner convenablement, en se désolidarisant visuellement des interactants immédiats par une orientation de regard vers un espace neutre, pour ré-ouvrir sa conversation privée dans cet espace public.

Il est dès lors difficile de rendre compte des normes de conduite qui régulent les usages des téléphones mobiles. Ce ne sont pas seulement les enregistrements vidéo décrits ici qui posent problème, mais plus généralement ce qu'ils donnent à voir. Ils rendent compte d'une réelle efficience des normes 
$11 \mathrm{LCN} \mathrm{n}^{\circ} / 2010$. Usages, pratiques et ergonomie des nouveaux supports numériques

de conduites sans permettre pour autant de les identifier et de les catégoriser. Il convient dès lors, en suivant une perspective ethnométhodologique, de parler d'une coordination entre les interactants impliqués d'où émerge une normalisation, circonstancielle et révisable, de leurs comportements respectifs. Cette coordination est pétrie d'implicite, d'échanges de regards ou de désengagements visuels de l'espace perceptif partagé. Nous allons maintenant décrire comment cette coordination visuelle des interactants peut s'opérer autour de la réception de la Mobile TV.

\section{L'encastrement des formes visuelles de la coordination sociale dans une forme de multi-activité}

Notre objectif consiste désormais à défendre l'hypothèse selon laquelle la malléabilité et l'aisance de mouvement offertes par les téléphones multimédia permettent aux utilisateurs d'inscrire leur réception de la Mobile TV dans des formes complexes de multi-activité (Datchary, Licoppe, 2007). La petite taille des écrans des téléphones leur permet de visionner un programme tout en percevant ce qui se joue en arrière-plan. Les usagers peuvent donc gérer, visuellement et simultanément, différentes activités, la réception et le déroulement du déplacement, tout en accordant une vigilance visuelle minimale aux multiples sollicitations extérieures produites par les co-présents. Cette convergence des orientations visuelles, liées à la gestion de ces activités dans les transports en commun, va nous permettre de documenter un mode de participation à la coordination sociale entre co-présents anonymes. Afin d'atteindre cet objectif, il convient de commencer par décrire comment les usages de la Mobile TV sont maintenus à la périphérie de l'espace perceptif partagé.

\section{Le positionnement de la réception télévisuelle nomade à la périphérie de l'espace perceptif partagé}

Il s'agit maintenant de décrire l'orientation visuelle adoptée par les utilisateurs pour visionner un programme de la Mobile TV dans un lieu public. Même si l'engagement visuel dans cette réception télévisuelle varie sensiblement, d'un usager à l'autre et d'une situation à l'autre, nous allons dégager une certaine régularité. A travers elle, nous montrerons comment cette réception s'inscrit dans le commerce des regards et affecte la nécessaire coordination avec les personnes environnantes. 
Pour atteindre cet objectif, il est pertinent de faire une photographie de cette orientation visuelle afin d'en décrire la structuration. Nous sélectionnons donc, parmi l'ensemble des prises de vues de la participante, celles qui nous paraissent être les plus représentatives. La première est extraite des premiers instants où elle regarde la série «Fame » à l'aide la Mobile TV (cf. 06.20.16; Photo 14), un peu plus de deux minutes après sa conversation téléphonique (04.00.21; cf. Photo 13). La deuxième a été sélectionnée de manière plus arbitraire. Elle représente son engagement visuel typique dans cette réception télévisuelle nomade (09.44.08; cf. Photo 15).

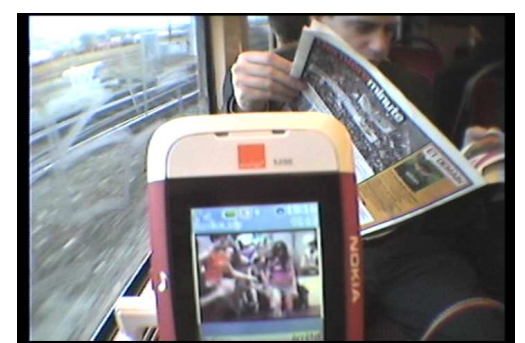

Photo 14 : Début de la réception

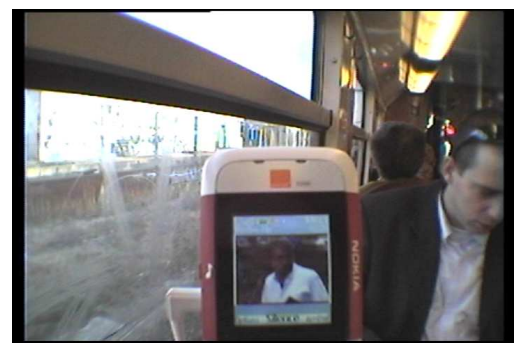

Photo 15 : Engagement visuel typique

Ces captures d'écran montrent que les utilisateurs positionnent l'écran au centre de leur champ de vision, à proximité immédiate de leur regard pour mieux percevoir ce qui se joue dans le cadre étroit de l'écran de leur téléphone. Cet aspect différencie les positionnements adoptés pour lire la presse et visionner la Mobile TV. On peut en effet observer que l'homme assis en face de la participante maintient son journal à une plus grande distance, notamment quand il le pose sur ses cuisses (cf. Photo 15). Il est probable que le port des lunettes caméra incite la participante à amplifier cette tendance. Mais elle reste bien réelle et récurrente dans la mesure où les programmes télévisés sont produits, et cadrés, pour être visionnés sur des écrans de plus en plus grands. Leur cadrage n'est pas optimisé pour les écrans des téléphones mobiles ce qui incite les utilisateurs à adopter cette posture aussi inconfortable qu'incongrue. Il apparait également que ce positionnement du téléphone et cette orientation visuelle amènent la participante à conserver le visage de l'homme dans son champ de vision. Ceci vaut lorsque sa tête est légèrement inclinée vers le bas (Photo 14) et lorsque sa tête est légèrement orientée vers l'extérieur (Photo 15).

Il apparait dès lors à quel point cette orientation du regard se démarque de celle qu'elle a adoptée précédemment, lorsqu'elle a rappelé son correspondant en regardant vers l'extérieur. Selon Murtagh, cette orientation vers un « espace neutre » est stratégique (Murtagh, 2001) car elle permet de maintenir dans la durée une activité intrusive en évitant d'être confronté aux regards désapprobateurs des personnes environnantes. Et, elle permet de manifester 
$13 \mathrm{LCN} \mathrm{n}^{\circ} / 2010$. Usages, pratiques et ergonomie des nouveaux supports numériques

une indisponibilité temporaire. On peut cependant considérer que ce positionnement stratégique n'est pas nécessairement déployé de manière réflexive, en réponse à une évaluation des coûts / bénéfices de telle ou telle posture, en ayant conscience de se désengager de l'espace perceptif commun au moment de tourner la tête vers l'extérieur. Il répond fortement aux circonstances pratiques et à la manière dont le commerce des regards se distribue dans un environnement cloisonné, soit plus précisément dans l'espace d'un wagon (Murtagh, 2001). La proximité et la promiscuité entre les passagers de cette rame du RER cloisonnent le champ visuel de cette utilisatrice, elles le rendent fortement perméable aux chevauchements de regards et le chassent vers l'extérieur, hors de l'espace perceptif partagé, là où la téléphoniste pourra entretenir sa conversation de manière plus sereine.

Il n'en va plus de même avec la Mobile TV. Sa réception est silencieuse et non intrusive. Il ne s'agit plus de décrire comment les téléphonistes jugulent la gêne, suscitée par leurs appels, en détournant stratégiquement leur regard. Il s'agit de comprendre comment ils maintiennent l'écran de leur téléphone et s'orientent visuellement dans cette réception télévisuelle par rapport à l'espace perceptif partagé pour ne pas éprouver de gêne et pouvoir visionner sereinement un programme. Comme les captures d'écran ci-dessus en témoignent, il semble que les usagers situent cette réception télévisuelle à la périphérie de l'espace perceptif partagé.

Pour comprendre ce positionnement, il convient de le rapprocher de la forme de courtoisie visuelle que Goffman a baptisée «inattention civile » (Goffman, 1963, p. 83 ; 1973). A travers cette notion, « Il soulignait ainsi qu’à la différence des interactions en face à face, qui requièrent une coordination permanente ainsi qu'une attention mutuelle forte basée sur une perception mutuelle, les relations en public se caractérisent par des formes de « courtoisie visuelle », qui, comme le coup d'œil, permettent aux passants par exemple de reconnaître la présence d'autrui, tout en acceptant son indétermination foncière » (Quéré, Relieu, 2001, p. 4). Cette courtoisie visuelle prend la forme de regards "non motivés », "non dirigés» (Sudnow, 2002) qui manifestent à autrui la prise en considération de sa présence sans l'investir pour autant d'une « curiosité spéciale» (Goffman, 1963, p. 84). Cette orientation du regard est, de ce fait, chargée d'une intention cordiale tant l'acteur s'applique «à faire semblant de s'occuper de ses propres affaires »(Goffman, 1973, p. 286). Cette inattention civile dissimule donc «une structure de collaboration» (Goffman, 1973), une réciprocité qui permet de rendre compte du positionnement des usages de la Mobile TV à la périphérie de l'espace perceptif partagé.

En effet, la participante adopte une orientation visuelle qui est clairement, voire ostensiblement, dirigée vers l'écran du téléphone, lui permettant de 
$14 \mathrm{LCN} \mathrm{n}^{\circ} / 2010$. Usages, pratiques et ergonomie des nouveaux supports numériques

visionner un programme tout en restant vigilante vis-à-vis de ce qui se joue autour d'elle, autour de l'écran. Dans la continuité de Goffman, il convient de ne pas limiter cet état de vigilance à la seule peur, à la surveillance des événements pouvant menacer son intégrité physique. Cet état lui permet avant tout de rester alerte en manifestant sa disposition à répondre aux éventuelles sollicitations extérieures. Son orientation du regard dans l'usage de la Mobile TV peut dès lors être rapprochée de la courtoisie visuelle de l'inattention civile dans la mesure où elle conserve un œil sur l'espace environnant, notamment sur l'homme assis face à elle, tout en invisibilisant la part d'attention qu'elle y accorde en publicisant son absorption dans une utilisation du téléphone mobile.

Il s'est agit ici de photographier l'orientation visuelle dans cet usage pour décrire son inscription dans les formes de la coordination sociale. En comparant cette orientation avec celle adoptée pour entretenir une conversation téléphonique, dans cette même situation, nous avons montré que la participante ne cherche pas à se substituer aux chevauchements de regards. Bien au contraire, la malléabilité et la petite taille des écrans des téléphones mobiles rendent ces usages perméables à la coordination avec les autres passagers ; cette perméabilité étant plus importante que celle ouverte par les pages encombrantes d'un quotidien. En convoquant cette perméabilité, il s'agit de dédramatiser les lectures pessimistes des comportements des téléphonistes en récusant l'idée d'un repli égoïste dans une bulle intimiste. Bien au contraire, en maintenant un engagement focalisé dans cette activité, les utilisateurs projettent un regard chargé de cordialité tant il paraît absorbé et dirigé vers un objet extérieur aux passagers les plus proches.

\section{Une coordination visuelle en prise avec une forme de multi-activité}

Nous venons de photographier l'engagement visuel adopté par les utilisateurs pour regarder la Mobile TV dans un transport en commun, en précisant qu'il se situait à la périphérie de l'espace perceptif partagé. Cette photographie permet de faire un arrêt sur image pour décrire en détail la structuration de cette orientation visuelle, en la comparant notamment avec l'« inattention civile » (Goffman, 1963, 1973). Il convient maintenant de retirer cette parenthèse en décrivant le déroulement des usages dans la durée afin d'observer comment l'orientation visuelle de la participante va évoluer, prenant alternativement pour objet l'écran du téléphone, les co-présents et les repères du déplacements.

Pour atteindre cet objectif, il est nécessaire d'affiner notre lecture de la coordination visuelle entre les passagers. Si les usages de la Mobile TV sont déployés à la périphérie de l'espace perceptif partagé, les frontières de cet 
$15 \mathrm{LCN} \mathrm{n}^{\circ} / 2010$. Usages, pratiques et ergonomie des nouveaux supports numériques

espace fluctuent dans le temps en fonction des changements d'orientations de regards des usagers et des personnes environnantes. Nous allons par exemple montré comment l'arrivée en gare incite les passagers à regarder en direction du quai, les amenant ainsi à chevaucher le regard des usagers de la Mobile TV. Les passagers renégocient ainsi leur participation dans l'espace perceptif partagé et amènent les utilisateurs à en faire de même. Il apparaît dès lors que l'espace interactionnel qui accueille ce commerce des regards ne doit pas être appréhendé de manière figé, tel un espace physique (Ciolek, Kendon, 1980 ; Kendon, 1990).

D'après Ciolek et Kendon, les interactants qui entretiennent une conversation définissent une « juridiction commune » par l'orientation de leurs « segments transactionnels» (les jambes, le buste, les bras, la tête, les yeux, etc.). Cet espace de l'interaction est dès lors «formé par un chevauchement continument maintenu entre les segments transactionnels des participants » (Ciolek, Kendon, 1980, p. 240). Ils se disposent ainsi pour se percevoir mutuellement afin de visualiser la gestuelle et les expressions faciales des uns et des autres. Il est intéressant de relier cette approche aux formes de coexistence dans les transports en commun.

En effet, ces lieux publics accueillent une forme de sociabilité singulière, se caractérisant par l'évitement des passagers (Kendon, 2001) dans la mesure où ils s'adressent rarement la parole et se coordonnent essentiellement à travers des échanges de regards. Il est alors intéressant de décrire comment les passagers déploient des stratégies d'évitement. L'inattention civile (Goffman, 1963, 1973) en est une mais cette courtoisie visuelle rend compte de croisements de regards éphémères et ne permet pas de rendre compte de cet évitement mutuel dans la durée. En effet, lorsque des passagers sont assis sur des sièges limitrophes des transports en commun, leurs segments transactionnels se chevauchent pendant plusieurs minutes. Ils sont dès lors amenés à les positionner de sorte à aménager un espace de coopération, une "juridiction commune» leur permettant d'atteindre leur but partagé : s'éviter, s'occuper de leurs propres affaires sans manifester une curiosité spéciale aux autres passagers. Ils doivent donc ré-agencer en permanence l'orientation de leurs segments transactionnels afin d'atteindre ce but commun et de le maintenir dans la durée. Nous considérons qu'ils doivent réviser en permanence leur participation dans cette zone d'inattention commune; le terme inattention renvoyant à la notion d'«inattention civile» (Goffman, 1963, 1973). L'objectif consiste à saisir comment les usages de la Mobile TV affectent la participation de l'utilisatrice dans cette zone d'inattention commune. Et, rétroactivement, comment l'engagement visuel dans cette réception télévisuelle est affecté par les 
$16 \mathrm{LCN} \mathrm{n}^{\circ} / 2010$. Usages, pratiques et ergonomie des nouveaux supports numériques

changements de positions des autres interactants engagés dans cette zone d'inattention commune.

Il est alors intéressant de noter comment la participante va orienter ses segments transactionnels une fois que l'homme, assis face à elle, va clore sa lecture du quotidien. En effet, une fois le RER arrêté à quai (11.08.00), il a plié en deux son journal et continue de le lire ainsi (11.14.01; cf. Photo 16). La participante lève quant à elle légèrement la tête tout en la tournant en direction du quai où une passante évolue (11.14.10 ; cf. Photo 17).

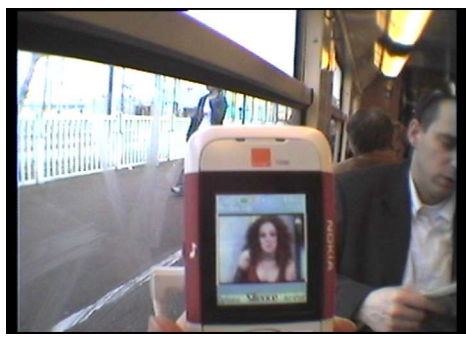

Photo 16. Arrivée en gare

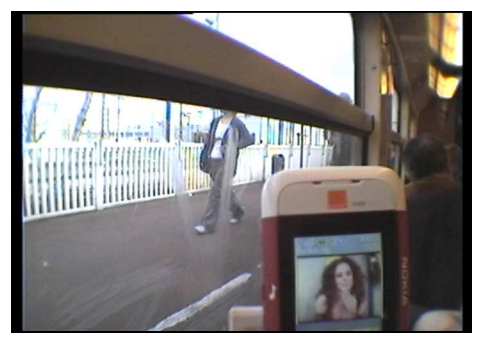

Photo 17. Modification de l'espace perceptif partagé

L'homme va faire de même. Lorsqu'il lève la tête et regarde vers le quai, ses segments transactionnels (S.T.) viennent chevaucher ceux de la participante (11.15.10 ; cf. Photo 18). Même s'il n'est pas possible de savoir ce que la participante regarde, il apparait comment son orientation visuelle est perméable à l'orientation du regard du passager assis face à elle. Il lui est bien plus difficile à cet instant de maintenir un lien visuel avec le programme télévisé sans être gênée par ce chevauchement de regards. Cette gêne peut être dégagée de son mouvement de tête vers la gauche qu'elle initie, quelques secondes après, tout en rapprochant davantage l'écran de ses yeux (11.15.24 ; cf. Photo 19).

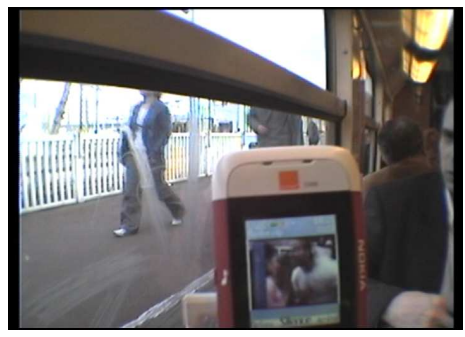

Photo 18. Chevauchement des S. T.

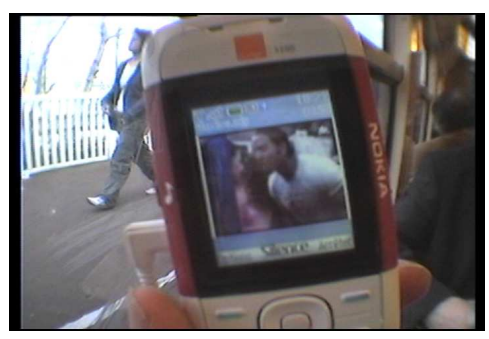

Photo 19. Evitement du chevauchement

Elle prolonge ensuite ce rapide mouvement de tête avant de le clore quand son regard est orienté vers l'extérieur (11.15.28 ; cf. Photo 20). Deux secondes après (11.17.24; cf. Photo 21), elle va incliner sa tête de sorte à ce que le panneau indiquant le nom de la station «Les Ardoines » entre dans son champ 
de vision. Cette inclination de tête nous permet de lui imputer l'intention de lire ce repère afin d'évaluer où elle en est dans ce trajet retour.

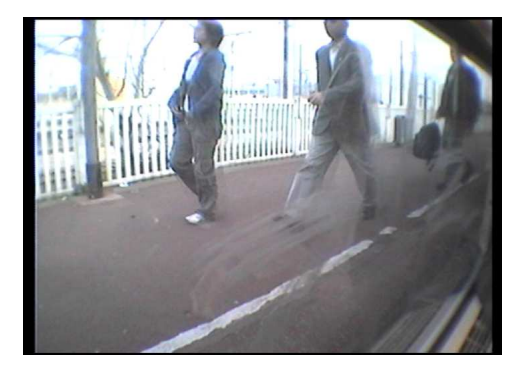

Photo 20. Fin du mouvement

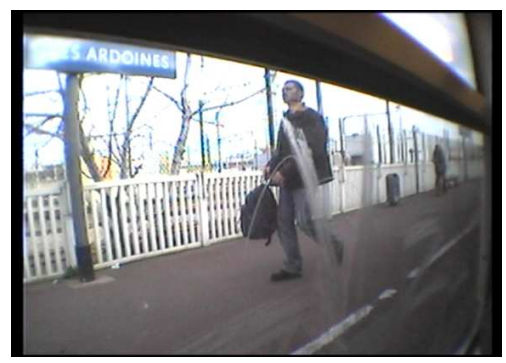

Photo 211. Regard dirigé vers un repère du déplacement

A travers cette séquence, il apparait clairement comment la gestion du déplacement peut être articulée avec la réception de la Mobile TV. La gestion de la mobilité, déléguée à un niveau périphérique de son attention, bascule ici au premier plan de ses préoccupations suite à une succession d'événements. Nous avons décrit le rôle de l'immobilisation du RER, les modifications de l'orientation du regard de l'homme assis face à elle, ainsi que les déambulations des passagers en arrière plan ou la présence du panneau indiquant le nom de la station. Il est intéressant de noter comment ces événements se présentent à son attention, en la désolidarisant peu à peu de la réception télévisuelle pour l'amener à adresser un regard dirigé sur un repère du déplacement. Ce déroulement des faits semble répondre à une certaine logique. Le RER ralenti peu à peu avant de marquer un arrêt en gare. Des passagers évoluent sur le quai. La participante regarde en direction du quai après qu'une passante soit entrée dans son champ de vision. Puis, elle baisse sa tête pour percevoir, sous la barre de la fenêtre du RER, le nom de la station. Cet enchaînement des faits répond à une certaine logique incitant à penser que ce cours d'action est traité de manière séquentielle. Chacune des phases qui le compose est prédictible. Et, chaque phase semble préfacer l'orientation future du cours d'action vers la phase suivante.

Cependant, cette séquence montre que la conduite de l'activité de déplacement, telle qu'elle est couplée ici à un usage de la Mobile TV, ne répond pas un tel traitement séquentiel. C'est l'enregistrement vidéo qui incite ici l'observateur extérieur à imputer, a posteriori, une logique à cette succession de faits. Une telle logique reviendrait à dire que chaque fois que l'utilisatrice rencontre cette succession de faits, elle se désengagerait visuellement de son usage pour surveiller, station après station, le déroulement de son trajet. Formulé en ces termes, il apparait clairement à quel point la gestion du 
$18 \mathrm{LCN} \mathrm{n}^{\circ} / 2010$. Usages, pratiques et ergonomie des nouveaux supports numériques

déplacement ne nécessite pas un tel investissement attentionnel. Comme nous l'avons dit précédemment, la taille et la malléabilité des téléphones mobiles permettent aux utilisateurs de visionner un programme tout en restant alerte vis-à-vis de ce qui se joue autour d'eux. L'habitué peut ainsi surveiller l'avancement de son trajet sans même y prêter garde, à un niveau périphérique de son attention. Mais, en experts, les utilisateurs exploitent cette succession d'événements comme des rappels à l'ordre, comme des déclencheurs leur rappelant de jeter des coups d'œil sur le déroulement de leur trajet. Autrement dit, ces contraintes et ces sollicitations extérieures contribuent à activer les coups d'œil sur la normalité des apparences chez celui qui se laisse capter par elles, chez celui qui est en état de «préoccupation» (Datchary, Licoppe, 2007). Celui qui gère de front plusieurs activités, comme un déplacement et un usage du téléphone mobile, s'installe dans cet état de préoccupation, notamment pour contrecarrer l'isolement sonore partiel dans lequel le port des écouteurs le plonge. En choisissant de visionner un programme pendant ce trajet, il s'engage à gérer de front ces deux activités. Il ne peut le faire qu'en déployant épisodiquement des coups d'œil sur l'avancement du trajet. C'est à travers eux qu'il va pouvoir se lever à temps, pour mener à bien sa mobilité, et qu'il va pouvoir baliser l'horizon temporel de l'activité de réception en anticipant le moment de l'arrivée en gare.

Il est dès lors préférable de parler ici d'une forme de multi-activité, où les activités réalisées simultanément vont être subordonnées les unes aux autres, alternativement en fonction des moments. Il y a multi-activité car les ressources mobilisées pour conduire l'activité de déplacement, comme le nom des stations, vont permettre de baliser le déroulement de l'usage de la Mobile TV. Et réciproquement, l'habitué qui a identifié les zones où le réseau téléphonique est disponible, et où il peut regarder la Mobile TV, saura où il se trouve sur son tronçon de trajet (Figeac, 2009). Cet encastrement des repères mobilisés pour conduire ces deux activités nous invite à parler d'une forme de multi-activité, la priorisation de l'une se traduisant par une suspension temporaire de la seconde et non par son interruption. C'est pourquoi, nous considérons que cette séquence ne permet pas de dire que cette succession d'événements participe à un traitement séquentiel de ces activités. Cette succession logique forme plutôt une ressource, un rappel à l'ordre incitant les utilisateurs à se désengager visuellement de la réception télévisuelle afin de suivre le déroulement de leur trajet, tout en écoutant le programme à l'aide des oreillettes.

Reste en suspend la question de l'encastrement des formes simples de la coordination sociale dans cette forme de multi-activité. Cette séquence est à ce titre particulièrement intéressante. Elle montre que la participante à adresser un regard dirigé vers un repère du déplacement (11.17.24 ; cf. Photo 21) juste après 
$19 \mathrm{LCN} \mathrm{n}^{\circ} / 2010$. Usages, pratiques et ergonomie des nouveaux supports numériques

que l'homme, situé face à elle, ait suspendu sa lecture d'un journal avant de regarder vers le quai (11.15.10; cf. Photo 18). A travers ce changement d'orientation, son regard est donc venu chevaucher celui de la participante qui a instantanément rapproché l'écran de ses yeux (11.15.24 ; cf. Photo 19) avant de se désengager visuellement de son usage en regardant à son tour le quai et le panneau indiquant le nom de la station (11.17.24 ; cf. Photo 21). Autrement dit, ce chevauchement des segments transactionnels, opéré par un mouvement de l'homme, a amené la participante à réajuster sa participation visuelle dans leur zone d'inattention commune. C'est donc ce chevauchement qui semble avoir incité l'utilisatrice à détourner son regard du téléphone avant d'adresser un regard dirigé vers un repère du déplacement. Ce réaménagement de la zone d'inattention commune a visiblement joué un rôle dans cette suspension de la réception au profit de la gestion du déplacement. Il apparaît donc que les formes visuelles de la coordination sociale s'encastrent à leur tour dans cette forme de multi-activité. Le processus de priorisation/subordination des activités conduites simultanément est lié à l'économie solidaire des «coups d'œil » et des « regards dirigés », à ces aménagements interactionnels de la zone d'inattention commune.

Ces aménagements répondent eux-aussi à une certaine prédictibilité, orchestrée et rendue intelligible à travers les "paramètres temporels de l'observation interpersonnelle » (Sudnow, 1972). Mais cette part de prédictibilité n'est pas suffisante pour avancer l'idée d'un enchaînement logique dans la distribution des engagements visuels entre la conduite des usages de la Mobile $\mathrm{TV}$, la gestion du déplacement dans un transport en commun et la coordination avec ceux qui peuplent ces lieux publics. Il parait plus pertinent de lier ces aménagements interactionnels à une forme de multi-activité. Cela permet de référer les changements d'orientation de regards à des déclencheurs (les chevauchements de regards, l'arrivée en gare, l'interruption d'un appel, etc.) sans pour autant les élever au rang de cause, générant par définition les mêmes effets. Ces déclencheurs n'activent pas un nouveau cours d'action, ils rendent simplement plus pertinente la priorisation du cours d'action qui était subordonné à ce moment-là et traité à un niveau périphérique de l’attention.

\section{Conclusion}

A travers cette contribution, nous avons tenté de décrire comment les usages de la Mobile TV s'inscrivent dans les formes simples de la coordination sociale (Conein, 2005). Les échanges de regards jouent un rôle central dans les modes de coordination entre des passagers anonymes des transports en commun car il est rare qu'ils rompent le silence en s'adressant la parole, si ce n'est pour s'adresser des excuses. A travers la description d'une conversation 
téléphonique d'une participante, il a été confirmé que cette prise de parole dans les transports en commun reste suffisamment intrusive pour inviter les téléphonistes à clore rapidement leurs conversations ou à détourner leurs regards de l'espace perceptif partagé pour éviter de croiser les regards désapprobateurs des autres passagers (Murtagh, 2001).

Il n'en va pas de même avec la réception télévisuelle nomade car elle reste silencieuse. Elle ne contraint pas les usagers à détourner leurs regards vers des « espaces neutres». Au contraire, elle paraît même chargée d'une intention cordiale, sur le modèle de la courtoisie visuelle baptisée « inattention civile » par Goffman, dans la mesure où elle permet aux usagers d'invisibiliser la part d'attention qu'ils accordent aux personnes environnantes. La participation à ces formes visuelles de la coordination sociale reste en dernière instance une affaire de subjectivité car chacun a la liberté de s'y soustraire. Si l'on se focalise sur le rôle des téléphones mobiles, en tant qu'intermédiaires technologiques qui affectent les modalités de participation à la vie publique, nous pouvons considérer qu'ils permettent de faire preuve de courtoisie visuelle. Ils sont suffisamment petits et malléables pour permettre de maintenir un usage focalisé et absorbant qui reste perméable à ce qui se joue autour de l'écran. Ils peuvent être maintenus de sorte à pouvoir réinvestir en un coup d'œil l'espace perceptif partagé sans jamais se désolidariser de la situation vécue et se replier dans une bulle intimiste asociale.

Nous avons également montré que l'articulation entre les usages de la Mobile TV et la conduite du déplacement ne peut pas être traitée sur le modèle de l'interruption. Idéalement, cela reviendrait à dire que les utilisateurs se désengagent de la réception télévisuelle lorsque le transport marque un arrêt, pour identifier où ils en sont dans leur parcours, avant de réinvestir leur écran. Cela reviendrait à dire que la coordination avec les interactants, qui nécessite de leur adresser des regards plus ou moins dirigés, pourrait être réalisée de manière totalement planifiable (projeter de surveiller la normalité des apparences au prochain arrêt ou à la prochaine coupure pub).

L'usager n'agit pas en marionnettiste. Il n'est ni caché par un décor, ni par une bulle qui lui permettrait de poursuivre sa réception sans subir les interférences des co-présents. Il ne distribue pas ses coups d'œil de manière planifiée en choisissant la totalité des moments où il va se désengager visuellement de sa réception. Il ne choisit pas nécessairement ceux où il va rechercher des indices sur l'avancement de son déplacement et ceux où il va être amené à se coordonner avec les autres passagers. C'est pourquoi cette activité médiatique s'inscrit dans une forme de multi-activité qui ne peut pas être rapprochée du modèle perception/action de la routine (Conein, Jacopin, 1994); ce modèle forme pourtant un implicite commun à de très nombreuses 
$21 \mathrm{LCN} \mathrm{n}^{\circ} / 2010$. Usages, pratiques et ergonomie des nouveaux supports numériques

études traitant des pratiques des nouveaux supports numériques dans les espaces publics.

La conduite de cette activité médiatique vient plutôt s'encastrer dans la gestion du déplacement dans un transport en commun et dans la coordination avec les passagers qui incombe à cette modalité de transport. Parler de multiactivité permet de montrer comment l'horizon temporel de la réception s'encastre dans celui du déplacement. Cela permet de donner sens à l'attention, aussi minimale soit-elle, accordée à ce qui se joue autour de l'écran. C'est à travers elle que ces deux activités s'encastrent et peuvent être menées à bien conjointement. Dès lors, le traitement de ces deux activités ne répond pas à une organisation séquentielle. Il n'y a pas d'interruption de l'une au profit de l'autre. Elles sont articulées, les orientations de l'une préfacent les orientations de l'autre. Elles sont toutes deux conduites de front avec une priorisation différente, de l'une par rapport à l'autre, suivant les moments.

Nous avons tenté ici de dégager le rôle de la coordination sociale dans cette forme de multi-activité. En choisissant de réaliser simultanément deux activités, la conduite du déplacement et la réception télévisuelle, les usagers se disposent à expérimenter des formes singulières de coordination avec les co-présents. Ils se désolidarisent partiellement de l'environnement auditif habité par les personnes à proximité. Ils font de même visuellement en maintenant l'écran du téléphone multimédia au centre de leur champ de vision. Ils se disposent ainsi à devoir redoubler de vigilance pour rester alerte vis-à-vis de ce qui se joue autour d'eux, notamment pour arriver à bon port. C'est ce qu'ils font en situant leur engagement visuel dans cette réception à la périphérie de l'espace perceptif partagé car ils peuvent visionner leur programme sans gêne tout en laissant leur réception perméable aux chevauchements de regards. Cette perméabilité peut les extraire de la quiétude de leur usage. Mais, elle leur permet avant tout de maintenir un état de "pré-occupation » (Datchary, Licoppe, 2007) en exploitant les orientations de regards des passagers à proximité comme des déclencheurs qui vont les rappeler à l'ordre en rendant pertinente la priorisation, temporaire, de l'activité de déplacement en adressant un coup d'œil sur l'avancement du trajet. Il s'agit d'une forme singulière d'« attention conjointe » (Conein, 2005, p.251) qui permet aux utilisateurs d'exploiter les phases où leurs segments transactionnels chevauchent ceux des personnes environnantes comme des déclencheurs les alertant des événements qu'elles ciblent visuellement, les incitant ainsi à surveiller la normalité des événements extérieurs et l'avancement du déplacement.

Notre contribution montre donc, par rapport à la polémique relative au manque de civilité des téléphonistes, que les usages de la Mobile TV sont profondément liés aux formes visuelles de la coordination sociale. Ce service 
télévisuel et, dans une certaine mesure, son support numérique permettent à l'utilisateur d'agencer avec une grande efficacité une zone qualifiée ici d'inattention commune; zone qui est par définition « civile». Ils permettent également, à celui qui le veut bien cette fois-ci, de participer aux échanges visuels de courtoisie. En revanche, ils poussent les utilisateurs à exploiter les interactants, avec qui ils aménagent une zone d'inattention commune, comme des rappels à l'ordre. Ils leur délèguent partiellement la tâche de surveiller la normalité des apparences, notamment en restant vigilants vis-à-vis des bruits sourds et des paroles qui viennent faire événement. Cet aspect des usages de la Mobile TV ne saurait alimenter la polémique relative à l'incivilité des téléphonistes. Il nous rappelle, comme nous l'avons montré dans la première partie, à quel point cette incivilité est liée à la propagation des bruits stridents des sonneries téléphoniques dans les lieux publics régulés par l'attente de comportements «calmes». Au fil de cette contribution, nous avons montré à quel point les interactants, investissant une zone d'inattention commune, et les co-présents, habitant visuellement un espace perceptif partagé, se coordonnent visuellement pour surveiller la normalité des apparences et s'occuper sereinement de leurs propres affaires. Nous pouvons dès lors considérer que cette incivilité des téléphonistes est profondément liée au fait que le retentissement des sonneries téléphoniques vient parasiter la projection des passagers dans l'orientation imminente et future de la situation vécue, opérée sans y accorder une attention particulière grâce aux "paramètres temporels de l'observation interpersonnelle» (Sudnow, 1972). De ce point de vue là, la multiplication des «notifications》 et des «alertes» sonores émises par les nouveaux supports numériques renforce la légitimité d'une telle approche, calme et dépassionnée, de leurs effets d'ordre proxémique. 
$23 \mathrm{LCN} \mathrm{n}^{\circ}$ /2010. Usages, pratiques et ergonomie des nouveaux supports numériques

\section{Bibliographie}

Conein B. (2005), Les sens sociaux, trois essais de sociologie cognitive, Paris, Economica.

Conein B., Jacopin E. (1994), "Action située et cognition : le savoir en place", Sociologie du travail, $\mathrm{n}^{\circ} 36,4$, pp. 475-500.

Ciolek M., Kendon A. (1980), "Environment and the Spatial Arrangement of Conversational Encounters", Sociological Inquiry, 50, 3-4, pp. 237 - 271.

Cui Y., Chipchase J., Jung Y. (2007), "Personal TV: A Qualitative Study of Mobile TV users", in Interactive TV: a Shared Experience, Springer Berlin / Heidelberg, pp. 195204.

Datchary C., Licoppe C. (2007), "La multi-activité et ses appuis : l'exemple de la "présence obstinée" des messages dans l'environnement de travail", @ ctivités, vol. 4, $\mathrm{n}^{\circ} 1$, pp. $4-29$.

Dey A. K., Salber D., Abowd G. D. (2001), "A conceptual framework and a toolkit for supporting the rapid prototyping of context-aware applications", IJHCS, 16.

Figeac J. (2009), "L'appropriation de la Mobile TV autour des réseaux de communication", Réseaux, n¹56, 2009.

Figeac J. (2010), "Vers une pragmatique de la pratique télévisuelle. Etude de l'appropriation de la Mobile TV en situation de mobilité", Permanence de la télévision, à paraître.

Goffman E. (1973), La mise en scène de la vie quotidienne - Les relations en public, Paris, Les Editions de Minuit.

Goffman E. (1963), Behaviour in public places. Notes on the social organization of gatherings, New York, Free Press.

Hall E.T. (1966), La dimension cachée, Seuil, 1978.

Jauréguiberry F. (1998), "Lieux publics, téléphone et civilité", Réseaux, n90.

Kendon A. (1990), Conducting Interaction. Patterns of behaviour in focused encounters, Cambridge University Press.

Knoche H., McCarthy J.D. (2005), "Good News for Mobile TV", Proceedings of WWRF14, 7-8 July 2005, San Diego, CA, USA.

Lehtola S., Mokka S. (2002), First steps of mobile digital television: state of the art and first user impressions, Research report TTE5-2002-18, VTT Information Technology.

Morel J. (2002), Une ethnographie de la téléphonie mobile dans les lieux publics, Réseaux, n $112-113$, p. 50-77.

Murtagh G. M. (2001), "Seeing the rules: preliminary observations of action, interaction and mobile phone use", in Wireless World. Social and interactional aspects of the mobile age, London, Springer-Verlag, 2002, p. 81-91. 
$24 \mathrm{LCN} \mathrm{n}^{\circ} / 2010$. Usages, pratiques et ergonomie des nouveaux supports numériques

O'Hara K., Mitchell A.S., Vorbau A. (2007), "Consuming Video on Mobile Devices", in Proceedings of CHI '07, April 28 - May 3, 2007, San Jose, California, USA.

Oksman V., Noppari E., Tammela A., Mäkinen M, Ollikainen V. (2007), News in Mobiles. Comparing text, audio and video, Research Notes 2375, VTT Tiedotteita.

Pecqueux A. (2009), " Embarqués dans la ville et la musique. Les déplacements préoccupés des auditeurs-baladeurs", Réseaux, n¹56.

Quéré L., Relieu M. (2001), Modes de locomotion et inscription spatiale des inégalités. Les déplacements des personnes atteintes de handicaps visuels et moteurs dans l'espace public. Rapport de recherche, PUCA.

Sacks H. (1992), "On exchanging glances", in Jefferson G., Lectures on conversation, vol. 1, Blackwell Publishers, Oxford, pp. 81-94.

Södergard C. (2003), Mobile television - technology and user experiences, Report on the Mobile-TV project, VT'T Information Technology.

Sudnow D. (1972), "Temporal parameters of interpersonal Observation", in Sudnow D., Studies in social interaction, The Free Press, New York, pp. 259 - 279.

Thibaud J-P. (1994), "Les mobilisations de l'auditeur-baladeur : une sociabilité publicative", Réseaux, nº 65, 1994, pp.71-83. 